0

doi: $10.5004 / \mathrm{dwt} .2019 .24363$

\title{
Enhanced boron rejection of a thin-film composite membrane by embedding additives including hydroxyl groups
}

\author{
Hee Ro Chae ${ }^{a}$, Deok-Ro Lee ${ }^{a}$, In-Chul Kim ${ }^{\mathrm{a}, *}$, Young-Nam Kwon ${ }^{\mathrm{b}, *}$ \\ ${ }^{a}$ Center for Membrane, Advanced Green Chemical Materials Division, Korea Research Institute of Chemical Technology, Daejeon 34114, \\ Republic of Korea, Tel. +82-42-860-7638; email: ickim@krict.re.kr(I.-C. Kim), Tel.+82 42-610-8592; email: chr0619@krict.re.kr (H.R. Chae), \\ Tel. +82 42-860-7040; email: shine9100@naver.com (D.-R. Lee) \\ ${ }^{b}$ School of Urban and Environmental Engineering, Ulsan National Institute of Science and Technology (UNIST), Ulsan 44919, \\ Republic of Korea, Tel. +82-52-217-2810; email: kwonyn@unist.ac.kr (Y.-N. Kwon)
}

Received 21 September 2018; Accepted 4 May 2019

\section{A B S T R A C T}

Herein, ( \pm )-3-amino-1,2-propandiol (APD) and N-methyl-D-glucamine (NMDG), which have hydroxyl groups, were respectively embedded in thin-film composite (TFC) membranes to enhance boron rejection. The membranes prepared with embedding materials (APD or NMDG) that are involved in interfacial polymerization showed an increased degree of crosslinking, which was confirmed by X-ray photoelectron spectrometry, causing a decrease of surface roughness and water flux. However, the APD and NMDG bound to the inside of the active layer enhanced boron rejection of the TFC membrane to $85.5 \%$ and $87.7 \%$, respectively, at $\mathrm{pH} 8$ due to tightening of membrane pores by the enhanced degree of crosslinking and complex formation of the additives with boric acid.

Keywords: Boron rejection; Reverse osmosis; Thin-film composite membrane

\footnotetext{
${ }^{*}$ Corresponding authors.
} 